\title{
Classical modeling of ultrafast coherent magneto-optical experiments
}

\author{
Y. Hinschberger and P.-A. Hervieux \\ Institut de Physique et Chimie des Matériaux de Strasbourg, CNRS and University of Strasbourg, 23 rue du Loess, \\ Boîte Postale 43, 67034 Strasbourg Cedex, France
}

(Received 18 June 2013; revised manuscript received 4 September 2013; published 15 October 2013)

\begin{abstract}
A classical model is developed for modeling ultrafast nonlinear coherent magneto-optical experiments performed on ferromagnetic thin films. Theoretical predictions of the Faraday rotation angles are compared to available experimental values and give meaningful insight into the physical mechanisms underlying the observed coherent magneto-optical phenomena. Under realistic conditions, the model successfully explains the observed trends. The crucial role played by the spin-orbit mechanism resulting from the direct interaction between the external electric field of the laser and the electron spins of the sample is underlined.
\end{abstract}

DOI: 10.1103/PhysRevB.88.134413

PACS number(s): 78.20.Ls, 78.47.jh, 75.78.Jp

\section{INTRODUCTION}

Since the discovery of the ultrafast demagnetization of a nickel film by a femtosecond laser pulse, ${ }^{1}$ study and ultimate control of ordered magnetic materials using ultrashort light pulses have been the object of intense investigations until becoming a full-fledged $\operatorname{art}^{2-4}$ given the diversity of experimental techniques implemented and the fundamental questions raised in the field of light-matter interactions.

Experimentally, the ultrafast demagnetization dynamics of ferromagnetic materials is usually investigated using a pump-probe time-resolved magneto-optical Kerr (TR-MOKE) configuration which consists in measuring the modified ellipticity $\eta$ and rotation angle $\theta$ of the reflected (Kerr geometry) or transmitted light beam (Faraday geometry). Indeed, in the framework of the linear magneto-optics the quantities $\eta$ and $\theta$ are proportional to the static magnetization of the sample and it is usually admitted that the spin-orbit interaction (SOI) is the microscopical mechanism which is responsible for the Faraday effect in ferromagnetic materials.

Moreover, in the nonlinear regime the modeling of magneto-optical experiments constitutes a very difficult theoretical challenge. First of all, the physical origin of the quick loss of magnetization is not fully resolved and remains unclear so far despite many relevant proposals: (i) spin-flip processes induced by scattering with phonons, ${ }^{5}$ magnons, ${ }^{6}$ or by angular momentum transfer with light; ${ }^{7}$ (ii) role played by the $\mathrm{SOI}^{8-10}$ or by the Coulomb interaction; ${ }^{11}$ (iii) coherent coupling between spins and photons ${ }^{12,13} \ldots$.

Unfortunately, $a b$ initio dynamical calculations leading to a macroscopic change of magnetization are very difficult to implement for such complex materials like ferromagnetic metals. Another current and crucial issue concerns the exact part of the magnetization change which is hidden in the parameters $\eta$ and $\theta$ corresponding to the total nonlinear anisotropy induced by the pump laser. ${ }^{14}$ In the past, several theoretical works based on sophisticated quantum mechanical models have provided nonlinear Kerr susceptibilities ${ }^{15-17}$ but none of them have really investigated the experimental magneto-optical parameters. Let us also stress the existence of an interesting, but complicated, approach to the nonlinear evolution of the Stokes parameters which can be found in Ref. 18 and a very recent work on the ultrafast magnetic dynamics of $\mathrm{NiO}$ where the nonlinear fluence dependence has been addressed in a nonperturbative way using a firstprinciples approach. ${ }^{19}$

Thus, whatever the model describing the magnetization dynamics, it seems important (and of fundamental interest) to provide an adequate modeling of the experimental observables $\eta$ and $\theta$ by using nonlinear anisotropic response functions. In this work, we propose to tackle this fundamental problem starting from the basic principles of magneto-optics and by working in the general framework of the Maxwell's equations. By considering nonlinear polarization currents, we derive nonlinear circular indices needed for the modeling of the nonlinear Faraday rotation angles. These latter quantities have been recently measured in a single-femtosecond-pulse Faraday experiment which was performed on a nickel film. ${ }^{12}$ In the same line as our work, it is worth mentioning recent theoretical studies devoted to the coherent photoexcitation of $(\mathrm{Ga}, \mathrm{Mn}) \mathrm{As}$ ferromagnetic semiconductors with linearly polarized light, where the interplay between the optical nonlinearities, the spin-orbit interaction, and the resulting optically induced magnetic anisotropy were treated microscopically. ${ }^{20-22}$

In the next section some basic considerations on linear magneto-optics are recalled and the principle of a single-femtosecond-pulse Faraday experiment is presented. In Sec. III, a nonlinear extension of the circular indices obtained from Maxwell's equations is described. A mean to incorporate the spin-orbit interaction using a macroscopic magneto-optical current is also introduced. Section IV is devoted to the theoretical modeling of the single-pulse Faraday experiment. Comparisons between theoretical predictions of the Faraday rotation angles and available experimental values of nickel thin film are performed in Sec. V. Finally, in Sec. VI, the results and the limitations of the theoretical modeling are discussed and some conclusions are given.

\section{LINEAR MAGNETO-OPTICS AND NONLINEAR FARADAY EXPERIMENT}

A linearly polarized light of pulsation $\omega$ propagating through a ferromagnetic layer of thickness $d$ is modified in a beam having an elliptical state of polarization characterized by the ellipticity $\eta$ and the rotation angle $\theta$. The complex phase $\Phi$ acquired during the propagation represents the phase shift between the right $(+)$ and the left $(-)$ circular states of 
polarization and is related to $\eta$ and $\theta$ as follows: ${ }^{4}$

$$
\Phi=\frac{\omega d}{2 c}\left(n_{+}-n_{-}\right) \equiv \theta+i \eta,
$$

where $c$ indicates the speed of light in vacuum and $n_{ \pm}$are the optical indices for the circular states $( \pm)$. In the polar configuration, where the effective magnetic field (molecular field, exchange...) acting inside the sample is oriented along the propagation direction $\mathbf{e}_{z}$ and the incident electric field belongs to the perpendicular plane defined by $\mathbf{e}_{x}$ and $\mathbf{e}_{y}$, the expressions of $n_{ \pm}$derived from the Maxwell's equations read

$$
n_{ \pm}^{2}=\epsilon_{x x} \pm i \epsilon_{x y},
$$

where $\epsilon_{x x}$ and $\epsilon_{x y}$ are, respectively, diagonal and nondiagonal elements of the dielectric tensor defined by $\mathbf{D}=\epsilon_{0} \mathbf{E}+\mathbf{P}=$ $\epsilon_{0}\left[\epsilon_{r}\right] \mathbf{E}$ and which can be written as

$$
\mathbf{D}=\epsilon_{0}\left[\begin{array}{ccc}
\epsilon_{x x} & \epsilon_{x y} & 0 \\
-\epsilon_{x y} & \epsilon_{x x} & 0 \\
0 & 0 & X
\end{array}\right] \mathbf{E} .
$$

In the above expression $X$ is anything. In Eq. (2), the condition $\epsilon_{x y} \ll \epsilon_{x x}$ is often used to transform $n_{ \pm}$and, by using Eq. (1), the Faraday phase appears to be proportional to the nondiagonal element of the dielectric tensor

$$
\Phi \approx \frac{i \omega d}{2 c} \frac{\epsilon_{x y}}{\sqrt{\epsilon_{x x}}},
$$

which can be expressed as a function of the effective magnetic field (or of the magnetization see in the following) thus breaking the symmetry of the medium.

The first expression of the tensor elements was given in 1908 by Voigt who considered the motion of charges induced by the Lorentz magnetic force. ${ }^{23,24}$ Within the framework of this first classical model, an agreement with the experimental results obtained on ferromagnetic materials can be found if a value of the internal magnetic field of the order of $10^{3} \mathrm{~T}$ is employed. It corresponds to the value of the Weiss molecular field.

Later in 1932, Hulme suggested that the spin-orbit interaction is needed to explain the coupling between optical properties of charges and the magnetization of the sample. ${ }^{25}$ He also pointed out that, because of the quenching of the orbital momentum in ferromagnetic materials, the effective field cannot affect the motion of charges. Then in 1955, Argyres developed a quantum mechanical model based on the SOI, leading to nondiagonal elements of the dielectric tensor proportional to the static magnetization of the system. ${ }^{26}$ Finally, let us mention two additional works which have successfully complemented the Argyres' model. ${ }^{27,28}$ Although these latter models are very powerful for describing magnetooptical effects in ferromagnetic materials, we here propose to work within the framework of the much simpler model of Drude-Voigt.

\section{A. The Drude-Voigt model}

In this model, matter is modeled as an ensemble of $N$ singleelectron atoms. The electron (of mass $m_{e}$ ) of each atomic site is described as a damped-harmonic oscillator bound to the nucleus by a Hooke force and feeling the Lorentz force. Thus, the equation of motion of an atomic bound electron is $(e>0)$

$$
\ddot{\mathbf{r}}+\frac{1}{\tau} \dot{\mathbf{r}}+\omega_{0}^{2} \mathbf{r}=-\frac{e}{m_{e}}\left(\mathbf{E}+\dot{\mathbf{r}} \wedge \mathbf{B}_{\text {mat }}\right),
$$

where $\mathbf{B}_{\text {mat }}=B_{\text {mat }} \mathbf{e}_{z}$ is the effective magnetic field inside the material and $\mathbf{E}$ is the electric field written as $\mathbf{E} \approx \mathbf{E}\left(e^{i \omega t}+\right.$ $e^{-i \omega t}$ ) (in the following, the same symbol is used to indicate a quantity in the direct or in the Fourier space). The motion of the electron labeled $i$ in the directions $\mathbf{e}_{x}$ and $\mathbf{e}_{y}$ generates an electric polarization field given by $\mathbf{p}_{i}=-e \mathbf{r}_{i}$ and the macroscopic dielectric polarization (in a volume $V$ ) is obtained by summing over all atomic sites $\mathbf{P} \equiv \frac{1}{V} \sum_{i}^{N}-e \mathbf{r}_{i}$. Working in the Fourier space with the mode $\omega$ and using the previous definition of $\mathbf{P}$, Eq. (5) may be written as

$$
\mathbf{P}=\chi \epsilon_{0} \mathbf{E}-i \xi \mathbf{P} \wedge \mathbf{e}_{z},
$$

where $\chi=\frac{\mathfrak{n} e^{2}}{m_{e} \epsilon_{0} D(\omega)}=\frac{\omega_{p}^{2}}{D(\omega)}$ with $D(\omega)=\left(\omega_{0}^{2}-\omega^{2}+\frac{i \omega}{\tau}\right)$ the linear electric susceptibility and $\xi=\frac{e \omega B_{\text {mat }}}{m_{e} D(\omega)}$ is the anisotropy parameter. Furthermore, $\mathfrak{n} \equiv N / V$ is the electron density and $\omega_{p}=\sqrt{\frac{\mathfrak{n} e^{2}}{m_{e} \epsilon_{0}}}$ is the plasmon pulsation.

The projection of Eq. (6) onto the $\mathbf{e}_{x}$ and $\mathbf{e}_{y}$ axes gives a system of two coupled equations for $P_{x}$ and $P_{y}$ which can be easily solved. The expression of the polarization vector reads

$$
\mathbf{P}=\epsilon_{0}\left[\begin{array}{ccc}
\frac{\chi}{1-\xi^{2}} & -\frac{i \xi \chi}{1-\xi^{2}} & 0 \\
\frac{i \xi \chi}{1-\xi^{2}} & \frac{\chi}{1-\xi^{2}} & 0 \\
0 & 0 & \chi
\end{array}\right] \mathbf{E} .
$$

Then, the dielectric permittivity tensor may be expressed as $\mathbf{D}=\epsilon_{0} \mathbf{E}+\epsilon_{0}[\chi] \mathbf{E}$ leading to the following expression of the tensor elements (3):

$$
\begin{aligned}
& \epsilon_{x x}=1+\frac{\chi}{1-\xi^{2}}, \\
& \epsilon_{x y}=-i \xi \frac{\chi}{1-\xi^{2}} .
\end{aligned}
$$

Thus, since the nondiagonal element of the dielectric tensor $\epsilon_{x y}$ is proportional (via the anisotropy parameter $\xi$ ) to the effective magnetic field inside the material $B_{\text {mat }}$, one concludes that the magneto-optical signal given by Eq. (4) is a measure of the magnetization of the sample (which is a part of the total internal magnetic field, $\mathbf{B}_{\text {mat }}$ ).

\section{B. Nonlinear Faraday experiment}

Unfortunately, in the nonlinear regime, the previous results cannot be used to model a magneto-optical signal since there is no dependence in the external electric field. In order to study this nonlinear dependence we propose to focus on a recent experimental work performed by Bigot et al. ${ }^{12}$ This team realized the following experiment: a 48 fs laser pulse is focused on a ferromagnetic $d=7.5 \mathrm{~nm}$ nickel thin film, and rotation and ellipticity are measured using a transmission configuration (Faraday configuration). The studies were performed over four orders of magnitude of the absorbed energy covering the range from $10^{-3}$ to $1 \mathrm{~mJ} / \mathrm{cm}^{2}$. It was observed that both rotation and ellipticity exhibit a nonlinear dependence in the absorbed energy (or in a similar manner in the external electric field). The setup of the experiment and the results mentioned above are summarized in Fig. 1. 


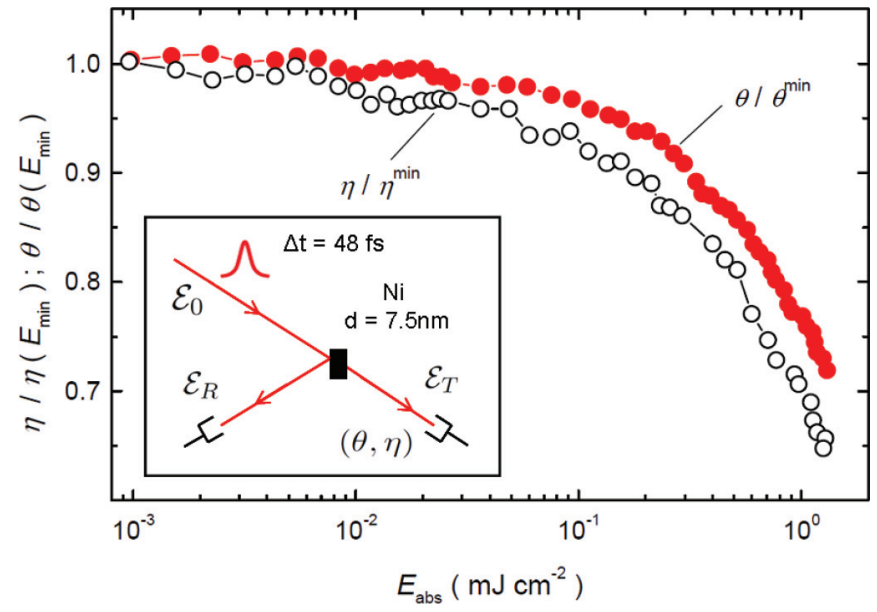

FIG. 1. (Color online) Principle of the single-pulse-like Faraday experiment performed in Ref. 12; normalized ellipticity and rotation as functions of the absorbed energy (see text).

We can estimate the amplitude $E$ of the laser electric field by noting that the total energy of the laser pulse is $\mathcal{E}_{0}=$ $\frac{c \epsilon_{0} \Delta t E^{2}}{2}$, where $\Delta t$ is the pulse duration. ${ }^{29}$ Furthermore, the absorbed energy $\mathcal{E}_{\text {abs }} \equiv \mathcal{E}_{A}$ is determined by the relation $\mathcal{E}_{0}=$ $\mathcal{E}_{R}+\mathcal{E}_{T}+\mathcal{E}_{A}$, where $\mathcal{E}_{0}, \mathcal{E}_{R}$, and $\mathcal{E}_{T}$ are, respectively, the incident, reflected, and transmitted light energies. According to the authors of, ${ }^{12}$ the absorption coefficient $\mathcal{A} \equiv \mathcal{E}_{A} / \mathcal{E}_{0}$ is constant over the entire energy range from $10^{-3}$ to $1 \mathrm{~mJ} / \mathrm{cm}^{2}$. For example, $\mathcal{E}_{0}=1.08 \mathrm{~mJ} / \mathrm{cm}^{2}, \mathcal{E}_{R}=0.35 \mathrm{~mJ} / \mathrm{cm}^{2}$, and $\mathcal{E}_{T}=0.31 \mathrm{~mJ} / \mathrm{cm}^{2}$ leads to $\mathcal{E}_{A}=0.42 \mathrm{~mJ} / \mathrm{cm}^{2}$ and $\mathcal{A}=0.39$. Finally, the relation between the absorbed energy $\mathcal{E}_{\text {abs }}$ and the amplitude $E$ of the laser field is given by

$$
\mathcal{E}_{0}=\frac{\mathcal{E}_{\mathrm{abs}}}{\mathcal{A}}=\frac{c \epsilon_{0} \Delta t E^{2}}{2} .
$$

Thus, the energy varying from $10^{-3}$ to $1 \mathrm{~mJ} / \mathrm{cm}^{2}$, it corresponds to an electric field amplitude between $10^{7}$ and $10^{9} \mathrm{~V} / \mathrm{m}$.

Because the SOI is the main mechanism responsible for the Faraday effect, the authors of Ref. 12 suggested that the observed nonlinear dependence of $\theta$ and $\eta$ with the incident electric field of the laser pulse can be associated to a SOI with the latter and the electron spins of the sample. It leads to a coherent magnetization change originating from a relativistic and direct interaction between spins and photons.

Without rejecting this microscopic interpretation, we here propose to look at the results of the above experiment from a more macroscopic point of view. The Faraday phase is interpreted as the phase shift between the circular states of light [see Eq. (1)]. The starting point is that the expression of the optical indices $n_{ \pm}^{2}$ of Eq. (2) leading to $\Phi \approx \frac{i \omega d}{2 c} \frac{\epsilon_{x y}}{\sqrt{\epsilon_{x x}}}$ is incomplete since there is no dependence in the laser electric field amplitude $E$. At high light intensities, the relative changes of $\theta=\operatorname{Re}(\Phi)$ and $\eta=\operatorname{Im}(\Phi)$ in Fig. 1 represent the total nonlinear anisotropy induced by the electric field. Therefore, the Faraday phase should be expressed in terms illustrating clearly both nonlinearities and the dependence with the amplitude of the electric field of the laser pulse.
In the next section, we propose to build a nonlinear expression of the optical indices involving the third-order electric susceptibility and the amplitude of the electric field.

\section{NONLINEAR EXTENSION}

This section is divided into three parts. (i) As the optical indices are related to the dielectric tensor elements, the first step is to derive the nonlinear third-order dielectric response. (ii) Then, from the associated dielectric current and the Maxwell equations, the nonlinear expressions for the optical indices are derived. (iii) The obtained results are completed by adding a macroscopic magneto-optical current originating from the microscopic SOI.

\section{A. Anharmonic Drude-Voigt model}

The nonlinear optical response is related to the microscopic interactions between the different particles in the material. A bound electron feels the influence of the other atomic sites and its potential energy becomes anharmonic $\mathcal{E} p=$ $\frac{m_{e} \omega_{0}^{2}}{2} r^{2}-\frac{m_{e} b}{4} r^{4}$ leading to a nonlinear Hooke force $\mathbf{F}=$ $-m_{e} \omega_{0}^{2} \mathbf{r}+m_{e} b \mathbf{r}^{3}{ }^{30}$ The equation of motion (5) is modified into

$$
\ddot{\mathbf{r}}+\frac{1}{\tau} \dot{\mathbf{r}}+\omega_{0}^{2} \mathbf{r}=b \mathbf{r}^{3}-\frac{e}{m_{e}}\left(\mathbf{E}+\dot{\mathbf{r}} \wedge \mathbf{B}_{\mathrm{mat}}\right) .
$$

The above equation can be solved using a perturbative development $\mathbf{r}(t)=\mathbf{r}^{(1)}(t)+\mathbf{r}^{(3)}(t)$, where $\mathbf{r}^{(1)}(t)$ and $\mathbf{r}^{(3)}(t)$ are, respectively, first- and third-order displacement vector solutions of the following equations:

$$
\begin{aligned}
\ddot{\mathbf{r}}^{(1)}+\frac{1}{\tau} \dot{\mathbf{r}}^{(1)}+\omega_{0}^{2} \mathbf{r}^{(1)} & =-\frac{e}{m_{e}}\left(\mathbf{E}+\dot{\mathbf{r}}^{(1)} \wedge \mathbf{B}_{\mathrm{mat}}\right), \\
\ddot{\mathbf{r}}^{(3)}+\frac{1}{\tau} \dot{\mathbf{r}}^{(3)}+\omega_{0}^{2} \mathbf{r}^{(3)} & =b\left(\mathbf{r}^{(1)}\right)^{3}-\frac{e \dot{\mathbf{r}}^{(3)}}{m_{e}} \wedge \mathbf{B}_{\text {mat }} .
\end{aligned}
$$

The above first equation is similar to Eq. (5) given in the previous section and has been already solved. Equation (11) is solved by injecting the solution of Eq. (5) in the term $b\left(\mathbf{r}^{(1)}\right)^{3}$. As the electric field is expressed as $\mathbf{E}(t) \approx \mathbf{E}\left(e^{i \omega t}+e^{-i \omega t}\right)$, the total first-order response is $\mathbf{r}^{(1)}=\mathbf{r}_{\omega}^{(1)} e^{i \omega t}+\mathbf{r}_{-\omega}^{(1)} e^{-i \omega t}$ with $\mathbf{r}_{\omega}^{(1)}=\frac{-e \mathbf{E}}{m_{e} D(\omega)}$. By taking the cube of $\mathbf{r}^{(1)}(t)$ one obtains four terms vibrating at $3 \omega, \omega,-\omega$, and $-3 \omega$. Due to the fact that here we only want to work with the $\omega$ mode, the term $b\left(\mathbf{r}^{(1)}\right)^{3}$ is replaced by $3 b\left(\mathbf{r}_{\omega}^{(1)}\right)^{2}\left(\mathbf{r}_{-\omega}^{(1)}\right) e^{i \omega t}$ and Eq. (11) becomes

$$
\ddot{\mathbf{r}}^{(3)}+\frac{1}{\tau} \dot{\mathbf{r}}^{(3)}+\omega_{0}^{2} \mathbf{r}^{(3)}=\frac{-3 m_{e}^{-3} b e^{3} \mathbf{E}^{3}}{D^{2}(\omega) D(-\omega)}-\frac{e \dot{\mathbf{r}}^{(3)}}{m_{e}} \wedge \mathbf{B}_{\mathrm{mat}} .
$$

The nonlinear term induces a microscopic third-order polarization vector $\mathbf{p}_{i}^{(3)}=-e \mathbf{r}_{i}^{(3)}$. The macroscopic dielectric polarization density is obtained by summing the microscopic polarization of each site $\mathbf{P}^{(3)}=\frac{1}{V} \sum_{i}^{N}-e \mathbf{r}_{i}^{(3)}$. Working in the Fourier space and using the previous expression, Eq. (12) is transformed into

$$
\mathbf{P}^{(3)}=\epsilon_{0} \chi^{(3)} \mathbf{E}^{3}-i \xi \mathbf{P}^{(3)} \wedge \mathbf{e}_{z},
$$

where $\chi^{(3)}=\frac{3 \mathfrak{n} b e^{3}}{m_{e}^{3} D^{2}(\omega) D(-\omega) \epsilon_{0}}$. As already mentioned, Eq. (13) contains a mixing between the two components of the 
polarization vector $P_{x}^{(3)}$ and $P_{y}^{(3)}$. The solved system gives

$$
\begin{aligned}
& P_{x}^{(3)}=\frac{\chi^{(3)} \epsilon_{0}}{1-\xi^{2}}\left(\mathbf{E}^{3}\right)_{x}-\frac{i \xi \epsilon_{0}}{1-\xi^{2}} \chi^{(3)}\left(\mathbf{E}^{3}\right)_{y}, \\
& P_{y}^{(3)}=\frac{\chi^{(3)} \epsilon_{0}}{1-\xi^{2}}\left(\mathbf{E}^{3}\right)_{y}+\frac{i \xi \epsilon_{0}}{1-\xi^{2}} \chi^{(3)}\left(\mathbf{E}^{3}\right)_{x},
\end{aligned}
$$

where $\left(\mathbf{E}^{3}\right)_{x}=\left(E_{x}^{2}+E_{y}^{2}+E_{z}^{2}\right) E_{x}$. Neglecting the contribution in the $\mathbf{e}_{z}$ direction and by writing $\epsilon_{x x x x}=\frac{\chi^{(3)}}{1-\xi^{2}}$ and $\epsilon_{x x x y}=-i \xi \epsilon_{x x x x}$, the third-order polarization density for the $\omega$ mode may be expressed as a tensor involving the electric field, $\mathbf{P}^{(3)}=\epsilon_{0}\left[\chi^{(3)}\left(E^{2}\right)\right] \mathbf{E}$, or more explicitly

$$
\mathbf{P}^{(3)}=\epsilon_{0}\left[\begin{array}{ccc}
\epsilon_{x x x x}\left(E_{x}^{2}+E_{y}^{2}\right) & \epsilon_{x x x y}\left(E_{x}^{2}+E_{y}^{2}\right) & 0 \\
-\epsilon_{x x x y}\left(E_{x}^{2}+E_{y}^{2}\right) & \epsilon_{x x x x}\left(E_{x}^{2}+E_{y}^{2}\right) & 0 \\
0 & 0 & X
\end{array}\right] \mathbf{E} .
$$

Let us note that the nonlinear parameter $b$ can be determined by assuming that linear and nonlinear Hooke forces are equal at the interatomic distance $a$. Therefore, equaling $m_{e} \omega_{0}^{2} a \approx m_{e} b a^{3}$ gives $b \approx \frac{\omega_{0}^{2}}{a^{2}} .31$

\section{B. Nonlinear indices}

We start from the Maxwell-Faraday and Maxwell-Ampère equations where the source terms are the displacement density current and the first- and third-order polarization density current $\mathbf{j}=\mu_{0} \frac{\partial}{\partial t}\left(\mathbf{P}^{(1)}+\mathbf{P}^{(3)}+\epsilon_{0} \mathbf{E}\right)$. Using the results of the previous sections total current density can be written as $\mathbf{j}=\mu_{0} \frac{\partial}{\partial t}\left(\epsilon_{0}\left[\epsilon^{\prime}\right] \mathbf{E}\right)$, where $\left[\epsilon^{\prime}\right]$ is the sum of the tensors given in Eqs. (3) and (14). These two Maxwell equations may be expressed in the Fourier space (for the $\omega$ mode) as

$$
\begin{aligned}
& \nabla \wedge \mathbf{E}=-\frac{\partial}{\partial t} \mathbf{B} \Rightarrow i \mathbf{k} \wedge \mathbf{E}=i \omega \mathbf{B}, \\
& \nabla \wedge \mathbf{B}=\mu_{0} \frac{\partial}{\partial t}\left(\epsilon_{0}\left[\epsilon^{\prime}\right] \mathbf{E}\right) \Rightarrow i \mathbf{k} \wedge \mathbf{B}=-i \omega \mu_{0} \epsilon_{0}\left[\epsilon^{\prime}\right] \mathbf{E} .
\end{aligned}
$$

We suppose that the electric field may be modeled by a plane wave having the following wave vector $\mathbf{k}=(0,0, k)$. Equations (15) lead to a system of coupled equations for the components of the magnetic and electric fields. Eliminating the magnetic field components and noting that $n=\frac{c k}{\omega}$ one gets

$$
\begin{aligned}
& n^{2} E_{x}=\left(\epsilon_{x x}^{\prime} E_{x}+\epsilon_{x y}^{\prime} E_{y}\right), \\
& n^{2} E_{y}=\left(-\epsilon_{x y}^{\prime} E_{x}+\epsilon_{x x}^{\prime} E_{y}\right),
\end{aligned}
$$

where the elements of the dielectric tensor read $\epsilon_{x x}^{\prime}=$ $\epsilon_{x x}+\epsilon_{x x x x}\left(E_{x}^{2}+E_{y}^{2}\right) \equiv A+B\left(E_{x}^{2}+E_{y}^{2}\right)$ and $\epsilon_{x y}^{\prime}=\epsilon_{x y}+$ $\epsilon_{x y y y}\left(E_{x}^{2}+E_{y}^{2}\right) \equiv a+b\left(E_{x}^{2}+E_{y}^{2}\right)$. The latest equations can be written as

$$
\begin{gathered}
\left(n^{2}-A\right) E_{x}=a E_{y}+B\left(E_{x}^{3}+E_{y}^{2} E_{x}\right)+b\left(E_{x}^{2} E_{y}+E_{y}^{3}\right), \\
\left(n^{2}-A\right) E_{y}=-a E_{x}+B\left(E_{y}^{3}+E_{x}^{2} E_{y}\right)-b\left(E_{x}^{3}+E_{y}^{2} E_{x}\right) .
\end{gathered}
$$

Dividing the first equation by $E_{x}$ and the second by $E_{y}$ (we assume that $E_{x} \neq 0$ and $E_{y} \neq 0$ ) allows us to obtain the fraction $\frac{E_{y}}{E_{x}}$ by two different ways. Equaling these two expressions leads to a second-order polynomial equation for the variable $X=\left(n^{2}-A\right)$ which reads

$$
X^{2}+\beta X+\gamma=0
$$

with the coefficients

$$
\begin{aligned}
\beta= & -2 B\left(E_{x}^{2}+E_{y}^{2}\right)-b\left(\frac{E_{y}^{3}}{E_{x}}-\frac{E_{x}^{3}}{E_{y}}\right), \\
\gamma= & a^{2}+B^{2}\left(E_{x}^{2}+E_{y}^{2}\right)^{2}-B b\left(\frac{E_{x}^{5}}{E_{y}}+E_{y} E_{x}^{3}-E_{y}^{3} E_{x}-\frac{E_{y}^{5}}{E_{x}}\right) \\
& -b^{2}\left(E_{x}^{4}+E_{y}^{4}+2 E_{x}^{2} E_{y}^{2}\right) .
\end{aligned}
$$

The formal resolution of the above equation gives two solutions for $X=\left(n^{2}-A\right)$ and consequently for the optical indices $n^{2}$,

$$
\begin{aligned}
n_{ \pm}^{2}= & A+B\left(E_{x}^{2}+E_{y}^{2}\right)+\frac{b}{2}\left(\frac{E_{y}^{3}}{E_{x}}-\frac{E_{x}^{3}}{E_{y}}\right) \\
& \pm i \sqrt{a^{2}-\frac{b^{2}}{4}\left(4 E_{x}^{4}+4 E_{y}^{4}+\frac{E_{y}^{6}}{E_{x}^{2}}+6 E_{x}^{2} E_{y}^{2}+\frac{E_{x}^{6}}{E_{y}^{2}}\right)} .
\end{aligned}
$$

In order to simplify the last equation, we use a symmetric assumption. Indeed, physics has to be the same whatever the electric field vector in the plane perpendicular to the propagation direction. Thus, one can choose a frame of reference where $E_{x} \approx E_{y} \approx E$. This assumption leads to $\left(E_{x}^{2}+\right.$ $\left.E_{y}^{2}\right) \approx 2 E^{2},\left(4 E_{x}^{4}+4 E_{y}^{4}+\frac{E_{y}^{6}}{E_{x}^{2}}+6 E_{x}^{2} E_{y}^{2}+\frac{E_{x}^{6}}{E_{y}^{2}}\right) \approx 16 E^{4}$, and $\left(\frac{E_{y}^{3}}{E_{x}}-\frac{E_{x}^{3}}{E_{y}}\right) \approx 0$. Finally, replacing $A, a, B$, and $b$ by their full expressions, the nonlinear indices may be written as

$$
n_{ \pm}^{2} \approx \epsilon_{x x}+2 \epsilon_{x x x x} E^{2} \pm i\left(\epsilon_{x y}^{2}-4 \epsilon_{x x x y}^{2} E^{4}\right)^{1 / 2} .
$$

We propose to use this last equation for describing circular optical indices of a nonlinear anisotropic medium. It is worth noting that Eq. (2) is recovered if one puts $E=0$ in Eq. (18).

\section{Spin-orbit interaction: From first-principles to Maxwell's equations}

Equation (18) may be completed in order to incorporate a magneto-optical term originating from the spin-orbit interaction. By calculating the microscopic quantum probability current density at second order in $1 / m_{e}$ one can build a macroscopic magneto-optical current which is directly related to the spin-orbit interaction. In order to do so, we first remind one of a few general considerations in quantum mechanics.

The well-known expression of the probability current density of an electron in the presence of an external magnetic field at first order in $1 / m_{e}(e>0)$ is ${ }^{32}$

$$
\begin{aligned}
\mathbf{j}^{(1)}= & \frac{i \hbar}{2 m_{e}}\left(\psi \nabla \psi^{\dagger}-\psi^{\dagger} \nabla \psi\right)+\frac{e}{m_{e}} \mathbf{A} \psi^{\dagger} \psi \\
& +\frac{\hbar}{2 m_{e}} \nabla \wedge\left(\psi^{\dagger} \boldsymbol{\sigma} \psi\right),
\end{aligned}
$$

where $\psi$ is a Pauli spinor and $\sigma$ are the Pauli matrices. When considering a system of $N$ electrons, the previous 
expression can be utilized to build a macroscopic charge current density which can be used as a source term in the Maxwell equations. Indeed, by summing over all electrons we get $\mathbf{j}_{\text {Maxwell }}=\sum_{i=1}^{N}-e \mathbf{j}_{i}^{(1)}$.

In Ref. 26, Argyres has shown that the first two terms of (19) can be associated to the polarization current density $\mathbf{j}_{\text {pol }}=\frac{\partial \mathbf{P}}{\partial t}=\frac{\partial\left(\chi_{\epsilon_{0}} \mathbf{E}\right)}{\partial t}$. By defining the magnetization as $\mathbf{M}=$ $\sum_{i=1}^{N} \frac{-e \hbar}{2 m_{e}}\left(\psi_{i}^{\dagger} \sigma \psi_{i}\right)$, one may also notice that the spin term of (19) leads to the magnetization current density $\mathbf{j}_{\text {mag }}=$ $\nabla \wedge \mathbf{M}$.

The probability current density can be derived from a variational principle using the Schrödinger-Pauli hamiltonian at first-order in $1 / m_{e}: H^{(1)}(\mathbf{A})=\frac{(\mathbf{p}+e \mathbf{A})^{2}}{2 m_{e}}-e \Phi+\frac{e \hbar}{2 m_{e}} \boldsymbol{\sigma} \cdot \mathbf{B}$, with $\mathbf{B}=\boldsymbol{\nabla} \wedge \mathbf{A}$. In Ref. 32, this work is performed by writing the elementary variation of the energy $\delta\langle H(\mathbf{A})\rangle \equiv$ $\int \psi^{\dagger} H(\delta \mathbf{A}) \psi d \tau$ with respect to $\delta \mathbf{A}$ and assuming the following relation of the electromagnetic energy:

$$
\int \psi^{\dagger} H^{(1)}(\delta \mathbf{A}) \psi d \tau \equiv-\int(-e) \mathbf{j}^{(1)}\left(\psi^{\dagger}, \psi\right) \cdot \delta \mathbf{A} d \tau .
$$

Therefore, the calculation of $H^{(1)}(\delta \mathbf{A})$ leads to the expression of $\mathbf{j}^{(1)}$. Here, we propose to extend this procedure up to the second order in $1 / m_{e}$ by using the nonrelativistic SchrödingerPauli Hamiltonian at second order in $1 / m_{e}$ which includes the spin-orbit interaction ${ }^{33,34}$

$$
\begin{aligned}
H^{(2)}= & \frac{(\mathbf{p}+e \mathbf{A})^{2}}{2 m_{e}}-e \Phi+\frac{e \hbar}{2 m_{e}} \boldsymbol{\sigma} \cdot \mathbf{B}+\frac{e \hbar^{2}}{8 m_{e}^{2} c^{2}} \nabla \cdot \mathbf{E} \\
& +\frac{e \hbar}{8 m_{e}^{2} c^{2}} \boldsymbol{\sigma} \cdot[\mathbf{E} \wedge(\mathbf{p}+e \mathbf{A})-(\mathbf{p}+e \mathbf{A}) \wedge \mathbf{E}] .
\end{aligned}
$$

Moreover, the calculation of $H^{(2)}(\delta \mathbf{A})$ will give terms involving $\partial_{t} \delta \mathbf{A}$ because of the presence of the electric field $\mathbf{E}=-\nabla \Phi-\partial_{t} \mathbf{A}$. Nevertheless one can neglect them by working in the Coulomb gauge $(\boldsymbol{\nabla} \cdot \mathbf{A}=0)$ and in the quasistatic approximation $\left[c^{2} \Delta \mathbf{A} \gg \partial_{t^{2}}^{2} \mathbf{A} \Leftrightarrow\left(c^{2} T\right) \delta \mathbf{A} \gg\right.$ $L^{2}\left(\partial_{t} \delta \mathbf{A}\right) \Leftrightarrow c \delta \mathbf{A} \gg L\left(\partial_{t} \delta \mathbf{A}\right)$ with $\left.(L=c T)\right]$. Indeed, the first assumption eliminates the influence of the Darwin term $\boldsymbol{\nabla} \cdot \delta \mathbf{E} \approx \partial_{t} \boldsymbol{\nabla} \cdot \delta \mathbf{A}=0$ and the second one shows that the term $\boldsymbol{\sigma} \cdot(\mathbf{E} \wedge \delta \mathbf{A})$ is more important than $\boldsymbol{\sigma} \cdot\left[\left(\partial_{t} \delta \mathbf{A}\right) \wedge \mathbf{A}\right]$ as $(\mathbf{E} \delta \mathbf{A}) \gg\left[\left(\partial_{t} \delta \mathbf{A}\right) \mathbf{A}\right]$ is equivalent to $c \delta \mathbf{A} \gg L\left(\partial_{t} \delta \mathbf{A}\right)$ with $E \approx c B \approx c A / L$.

Thus, in the Coulomb gauge and in the quasistatic approximation, using the Hamiltonian of Eq. (21), Eq. (20) at second order in $1 / m_{e}$ reads

$$
\begin{aligned}
& \int \psi^{\dagger}\left(\frac{e}{2 m_{e}}(\mathbf{p} \cdot \delta \mathbf{A}+\delta \mathbf{A} \cdot \mathbf{p})+\frac{e^{2}}{m_{e}} \mathbf{A} \cdot \delta \mathbf{A}\right. \\
& \left.+\frac{e \hbar}{2 m_{e}} \boldsymbol{\sigma} \cdot(\nabla \wedge \delta \mathbf{A})+\frac{e^{2} \hbar}{4 m_{e}^{2} c^{2}} \sigma \cdot(\mathbf{E} \wedge \delta \mathbf{A})\right) \psi d \tau,
\end{aligned}
$$

which can be easily transformed by integrating by part and using $\mathbf{p}=-i \hbar \nabla$ (see also Ref. 32) as

$$
\begin{aligned}
& \int e \delta \mathbf{A} \cdot\left(\frac{i \hbar}{2 m_{e}}\left(\psi \nabla \psi^{\dagger}-\psi^{\dagger} \nabla \psi\right)+\frac{e}{m_{e}} \psi^{\dagger} \psi \mathbf{A}\right. \\
& \left.+\frac{\hbar}{2 m_{e}} \nabla \wedge\left(\psi^{\dagger} \boldsymbol{\sigma} \psi\right)+\frac{e \hbar}{4 m_{e}^{2} c^{2}}\left(\psi^{\dagger} \boldsymbol{\sigma} \psi \wedge \mathbf{E}\right)\right) d \tau .
\end{aligned}
$$

From the above equation one can deduce the probability current density which includes the correction at second order in $1 / m_{e}$ due to the spin-orbit interaction ${ }^{35}$

$$
\mathbf{j}=\mathbf{j}^{(1)}+\frac{e \hbar}{4 m_{e}^{2} c^{2}}\left(\psi^{\dagger} \boldsymbol{\sigma} \psi \wedge \mathbf{E}\right)+\vartheta\left(1 / m_{e}^{3}\right) .
$$

Defining the magnetization as $\mathbf{M}=\sum_{i=1}^{N} \frac{-e \hbar}{2 m_{e}}\left(\psi_{i}^{\dagger} \boldsymbol{\sigma} \psi_{i}\right)$ and by noting $\alpha=\frac{e}{2 m_{e} c^{2}}$ one can build a macroscopic Maxwell's charge current with a new term $\mathbf{j}_{\text {mo }}$ having a magneto-optical vectorial form and originating directly from the microscopic spin-orbit interaction

$$
\mathbf{j}_{\text {Maxwell }}=\sum_{i=1}^{N}-e \mathbf{j}_{i} ;=\frac{\partial \mathbf{P}}{\partial t}+\nabla \wedge \mathbf{M}+\underbrace{\alpha \mathbf{M} \wedge \mathbf{E}}_{\mathbf{j}_{m o}} .
$$

Thus, we propose to add this term to the expression of the nonlinear indices. Moreover, in a nonlinear medium, the electric field inside the material is the sum of the external electric field and the first- and third-order polarization fields. Indeed, the magneto-optical current has to be completed by the following expression:

$$
{ }^{n l} \mathbf{j}_{m o}=\alpha \mathbf{M} \wedge\left(\mathbf{E}+\frac{\mathbf{P}}{\epsilon_{0}}+\frac{\mathbf{P}^{(3)}}{\epsilon_{0}}\right)=\frac{\alpha \mathbf{M}}{\epsilon_{0}} \wedge\left(\epsilon_{0}\left[\epsilon^{\prime}\right] \mathbf{E}\right),
$$

where $\left[\epsilon^{\prime}\right]$ is the tensor discussed in Sec. III B. Using this expression in the Maxwell-Ampère equation, Eqs. (15) are modified as follows:

$$
\begin{aligned}
i \mathbf{k} \wedge \mathbf{E} & =i \omega \mathbf{B}, \\
i \mathbf{k} \wedge \mathbf{B} & =-\mu_{0} \epsilon_{0} i \omega\left(\left[\epsilon^{\prime}\right] \mathbf{E}+\frac{i \alpha \mathbf{M}}{\omega \epsilon_{0}} \wedge\left[\epsilon^{\prime}\right] \mathbf{E}\right) .
\end{aligned}
$$

By assuming a magnetization along the $z$ axis, $\mathbf{M}=M_{z} \mathbf{e}_{z}$, the elimination of the magnetic components leads to the following equations:

$$
\begin{aligned}
& n^{2} E_{x}=\left(\epsilon_{x x}^{\prime}+\lambda \epsilon_{x y}^{\prime}\right) E_{x}+\left(\epsilon_{x y}^{\prime}-\lambda \epsilon_{x x}^{\prime}\right) E_{y}, \\
& n^{2} E_{y}=\left(\epsilon_{x x}^{\prime}+\lambda \epsilon_{x y}^{\prime}\right) E_{y}-\left(\epsilon_{x y}^{\prime}-\lambda \epsilon_{x x}^{\prime}\right) E_{x},
\end{aligned}
$$

where $\lambda=\frac{i \alpha M_{z}}{\omega \epsilon_{0}}=\frac{i e M_{z}}{2 m_{e} c^{2} \omega \epsilon_{0}}$ represents the modifications carried out by the spin-orbit interaction in Eq. (16). The resolution of Eq. (23) is the same as the one used to solve Eq. (16). The solution is obtained by substituting $A=\left(\epsilon_{x x}+\lambda \epsilon_{x y}\right), a=$ $\left(\epsilon_{x y}-\lambda \epsilon_{x x}\right), B=\left(\epsilon_{x x x x}+\lambda \epsilon_{x x x y}\right)$, and $b=\left(\epsilon_{x x x y}-\lambda \epsilon_{x x x x}\right)$ in the expressions of $n_{ \pm}^{2}$ given by Eq. (17).

Finally, using the approximative solution of Eq. (18), we obtain the expression of the nonlinear indices with the magneto-optical corrections as

$$
\begin{aligned}
n_{ \pm}^{2} \approx & \left(\epsilon_{x x}+\lambda \epsilon_{x y}\right)+2\left(\epsilon_{x x x x}+\lambda \epsilon_{x x x y}\right) E^{2} \\
& \pm i\left[\left(\epsilon_{x y}-\lambda \epsilon_{x x}\right)^{2}-4\left(\epsilon_{x x x y}-\lambda \epsilon_{x x x x}\right)^{2} E^{4}\right]^{1 / 2} .
\end{aligned}
$$

The above expression improves Eq. (18) and represents an alternative way to incorporate the SOI in the circular optical indices.

\section{THEORETICAL MODELING OF THE SINGLE-PULSE FARADAY EXPERIMENT}

In this section we propose to model the single-pulse Faraday experiment of Ref. 12 presented in Sec. II. The Faraday phase 
propagating through the nickel film is supposed to be defined by the following relation:

$$
\Phi \equiv \Phi(E)=\theta+i \eta=\frac{\omega_{l} d}{2 c}\left[n_{+}(E)-n_{-}(E)\right],
$$

where $n_{ \pm}(E)$ is given by Eq. (18) or by Eq. (24). In order to compare with the experimental values depicted in Fig. 1, normalized rotation and ellipticity parameters are calculated using

$$
\begin{aligned}
& \theta / \theta^{\min }=\operatorname{Re}\left[\Phi\left(\mathcal{E}_{\text {abs }}\right)\right] / \operatorname{Re}\left[\Phi\left(\mathcal{E}_{\text {abs }}^{\min }\right)\right] \\
& \eta / \eta^{\min }=\operatorname{Im}\left[\Phi\left(\mathcal{E}_{\text {abs }}\right)\right] / \operatorname{Im}\left[\Phi\left(\mathcal{E}_{\text {abs }}^{\min }\right)\right] .
\end{aligned}
$$

In Ref. 12, the 48 fs laser pulse is centered at $\lambda_{l}=799$ $\mathrm{nm}$ leading to a central laser pulsation $\omega_{l}=\frac{2 \pi c}{\lambda_{l}}=2.36 \times$ $10^{15} \mathrm{rad} / \mathrm{s}$. The sample is a nickel thin film having a thickness of $d=7.5 \mathrm{~nm}$. The relation between the amplitude of the electric field $E$ and the absorbed energy $\mathcal{E}_{\text {abs }}$ is obtained from the formula given in Sec. II, namely, $\frac{c \epsilon_{0} E^{2}}{2}=\frac{\mathcal{E}_{\text {abs }}\left(\mathrm{mJ} / \mathrm{cm}^{2}\right) \times 10}{0.388 \times 48 \times 10^{-15}}$. In order to check the importance of the magneto-optical parameters, the study will be first performed using Eq. (18) and then using Eq. (24). To complete the study it remains to evaluate the dielectric tensor elements $\epsilon_{x x}, \epsilon_{x y}, \epsilon_{x x x x}$, and $\epsilon_{x x x y}$. In order to do so, and since the most important issue addressed in this work is the nonlinear dependence of the signal with the amplitude of the electric field [see Eqs. (18) and (24)], we propose to evaluate the dielectric response functions within a rather simple theoretical framework: the anharmonic Drude-Voigt model.

\section{A. Classical description of nickel}

In this part we develop a classical model for describing metallic and ferromagnetic properties of nickel. Let us recall that the nickel atom has the following electronic structure: $[\mathrm{Ar}] 4 s^{2} 3 d^{8}$. The nonlinear properties in the material are related to the bound electrons, and the conductivity properties to the free charge carriers.

The assumptions used in the present modeling are the following:

(i) According to Refs. 36 and 37, there is 0.6 conducting electron per atomic nickel site. We assume that the valence electronic shell can be modeled by one electron which is screened by the nucleus and the other core electrons. One fraction $x_{b}$ of this electron is considered to be bound and another fraction $x_{f}$ is considered to be free with $x_{b}+x_{f}=1$ [Fig. 2(a)].

(ii) The microscopic first- and third-order displacements of the bound and free charges $\left(\mathbf{r}_{b}^{(1)}, \mathbf{r}_{f}^{(1)}, \mathbf{r}_{b}^{(3)}, \mathbf{r}_{f}^{(3)}\right)$ are determined using the anharmonic Drude-Voigt model and lead to the macroscopic polarization densities $\left(\mathbf{P}_{b}^{(1)}, \mathbf{P}_{f}^{(1)}, \mathbf{P}_{b}^{(3)}, \mathbf{P}_{f}^{(3)}\right)$ with $\mathbf{P}_{a}^{(k)}=\frac{1}{V} \sum_{i}^{N_{a}}-e \mathbf{r}_{a_{i}}^{(k)}$, where $a=b, f$ and $k=1,3$.

(iii) The total electric field acting on the charges inside the material is the sum of the external laser electric field and the polarization field generated by the charges. Moreover, we assume that the free electrons dynamics is influenced by the bound charges dynamics, but not the contrary [Fig. 2(b)].

(iv) Finally, the effective magnetic field inside the material is noted $\mathbf{B}_{\text {mat }}$ and corresponds to the Weiss molecular field. (a)
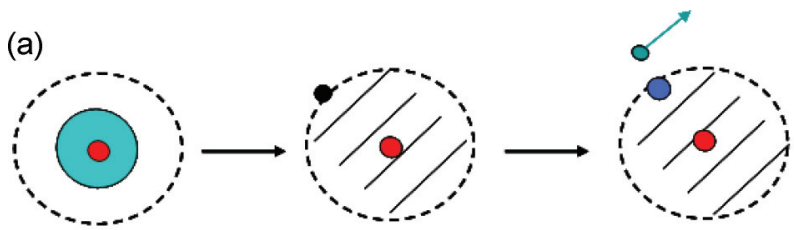

$0 \mathrm{xb} O+\mathrm{xf}$

(b)
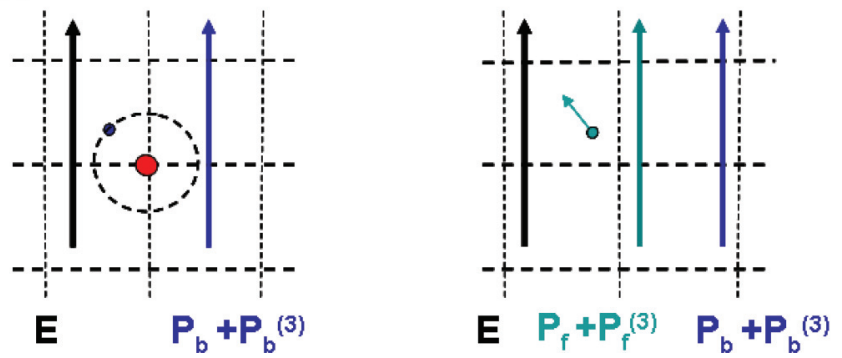

FIG. 2. (Color online) (a) Zener representation of a ferromagnetic material (see text). (b) Laser and polarization electric fields acting on bound and free electrons.

Thus, the dielectric tensor elements are determined by calculating the following quantity:

$$
\begin{aligned}
\mathbf{D} & =\epsilon_{0} \mathbf{E}+\mathbf{P}_{b}^{(1)}+\mathbf{P}_{f}^{(1)}+\mathbf{P}_{b}^{(3)}+\mathbf{P}_{f}^{(3)} \\
& =\epsilon_{0}\left(1+\left[\chi_{b}^{(1)}\right]+\left[\chi_{f}^{(1)}\right]+\left[\chi_{b}^{(3)}\left(E^{2}\right)\right]+\left[\chi_{f}^{(3)}\left(E^{2}\right)\right]\right) \mathbf{E} .
\end{aligned}
$$

Let us stress that our description of $\mathrm{Ni}$ is rather crude mainly due to the fact that in the effective-mass picture one neglects many-electron correlations and that the decomposition in free and bound electrons is performed more or less artificially. Here, a fully ab initio calculation including correlation effects would normally be necessary. ${ }^{19}$ However, as shown in the following, despite their weaknesses all these approximations lead to interesting results. Furthermore, due to its simplicity (which is not the case for first-principles models) this model might be useful for experimentalists for analyzing their data.

\section{B. Dielectric matter-bound electrons}

The equation of motion is almost identical to Eq. (9) of Sec. III A; we have just added the contribution of the polarization field and write $\tau_{b}$ for the damping associated to the bound electrons. It leads to

$$
\begin{aligned}
\ddot{\mathbf{r}}_{b} & +\frac{1}{\tau_{b}} \dot{\mathbf{r}}_{b}+\omega_{0}^{2} \mathbf{r}_{b} \\
& =-\frac{e \mathbf{E}}{m_{b}}-\frac{e}{m_{b}} \dot{\mathbf{r}}_{b} \wedge \mathbf{B}_{\text {mat }}+b \mathbf{r}_{b}^{3}-\frac{e}{m_{b} \epsilon_{0}}\left(\mathbf{P}_{b}^{(1)}+\mathbf{P}_{b}^{(3)}\right) .
\end{aligned}
$$

As before, the above equation is split into two different ones with $\mathbf{r}_{b}(t)=\mathbf{r}_{b}^{(1)}(t)+\mathbf{r}_{b}^{(3)}(t)$ :

$$
\begin{aligned}
& \ddot{\mathbf{r}}_{b}^{(1)}+\frac{1}{\tau_{b}} \dot{\mathbf{r}}_{b}^{(1)}+\omega_{0}^{2} \mathbf{r}_{b}^{(1)}=-\frac{e \mathbf{E}}{m_{b}}-\frac{e \mathbf{P}_{b}^{(1)}}{m_{b} \epsilon_{0}}-\frac{e \dot{\mathbf{r}}_{b}^{(1)}}{m_{b}} \wedge \mathbf{B}_{\mathrm{mat}}, \\
& \ddot{\mathbf{r}}_{b}^{(3)}+\frac{1}{\tau_{b}} \dot{\mathbf{r}}_{b}^{(3)}+\omega_{0}^{2} \mathbf{r}_{b}^{(3)}=b\left(\mathbf{r}_{b}^{(1)}\right)^{3}-\frac{e \mathbf{P}_{b}^{(3)}}{m_{b} \epsilon_{0}}-\frac{e \dot{\mathbf{r}}_{b}^{(3)}}{m_{b}} \wedge \mathbf{B}_{\mathrm{mat}} .
\end{aligned}
$$

By working in the Fourier space for the $\omega$ mode, by summing over all the $N_{b}$ bound electrons, and by defining $\mathfrak{n}_{b}=N_{b} / V$, 
allows us to transform the two latest equations into

$$
\begin{aligned}
& \mathbf{P}_{b}^{(1)}=\epsilon_{0} \chi_{b}^{(1)} \mathbf{E}-i \xi_{b} \mathbf{P}_{b}^{(1)} \wedge \mathbf{e}_{z}, \\
& \mathbf{P}_{b}^{(3)}=\epsilon_{0} \chi_{b}^{(3)} \mathbf{E}^{3}-i \xi_{b} \mathbf{P}_{b}^{(3)} \wedge \mathbf{e}_{z},
\end{aligned}
$$

where $\quad \chi_{b}^{(1)}=\frac{\mathfrak{n}_{b} e^{2}}{m_{b} \epsilon_{0} D_{b}(\omega)}, \quad \xi_{b}=\frac{e \omega B_{\text {mat }}}{m_{b} D_{b}(\omega)}, \quad \chi_{b}^{(3)}=\frac{3 \mathfrak{n}_{b} b e^{4}}{m_{b}^{3} D_{b}^{2}(\omega) D_{b}(-\omega)}$, and $D_{b}(\omega)=\left(\omega_{0}^{2}-\omega^{2}-\frac{\mathfrak{n}_{b} e^{2}}{m_{b} \epsilon_{0}}+\frac{i \omega}{\tau_{b}}\right)$ is the bound electron response function. Thus, by writing $\alpha_{b}^{(1)}=\frac{\chi_{b}^{(1)}}{1-\xi_{b}^{2}}$ and $\alpha_{b}^{\prime(3)}=$ $\frac{\chi_{b}^{(3)}}{1-\xi_{b}^{2}}\left(E_{x}^{2}+E_{y}^{2}\right)$, the first- and third-order macroscopic polarization density tensor for bound electrons may be written as

$$
\begin{aligned}
& \mathbf{P}_{b}^{(1)}=\epsilon_{0} \alpha_{b}^{(1)}\left[\begin{array}{ccc}
1 & -i \xi_{b} & 0 \\
i \xi_{b} & 1 & 0 \\
0 & 0 & X
\end{array}\right] \mathbf{E}, \\
& \mathbf{P}_{b}^{(3)}=\epsilon_{0} \alpha_{b}^{(3)}\left[\begin{array}{ccc}
1 & -i \xi_{b} & 0 \\
i \xi_{b} & 1 & 0 \\
0 & 0 & X
\end{array}\right] \mathbf{E} .
\end{aligned}
$$

\section{Metallic matter-conduction electrons}

For the free electrons, the equations of motion are slightly different. Indeed, there is no Hooke force, the damping factor is noted $\tau_{f}$, and the total electric field is the sum of the external laser field plus all the polarization fields:

$$
\begin{aligned}
\ddot{\mathbf{r}}_{f} & +\frac{1}{\tau_{f}} \dot{\mathbf{r}}_{f} \\
& =-\frac{e \mathbf{E}}{m_{f}}-\frac{e \dot{\mathbf{r}}_{f}}{m_{f}} \wedge \mathbf{B}_{\mathrm{mat}}-\frac{e}{m_{f} \epsilon_{0}}\left(\mathbf{P}_{b}^{(1)}+\mathbf{P}_{f}^{(1)}+\mathbf{P}_{b}^{(3)}+\mathbf{P}_{f}^{(3)}\right) .
\end{aligned}
$$

Here, the nonlinear character of the equation is introduced through the nonlinear polarization $\mathbf{P}_{b}^{(3)}$. Thus, by writing $\mathbf{r}_{f}(t)=\mathbf{r}_{f}^{(1)}(t)+\mathbf{r}_{f}^{(3)}(t)$ we get the two following equations:

$$
\begin{aligned}
& \ddot{\mathbf{r}}_{f}^{(1)}+\frac{1}{\tau_{f}} \dot{\mathbf{r}}_{f}^{(1)}=-\frac{e \mathbf{E}}{m_{f}}-\frac{e \mathbf{P}_{f}^{(1)}}{m_{f} \epsilon_{0}}-\frac{e \mathbf{P}_{b}^{(1)}}{m_{f} \epsilon_{0}}-\frac{e \dot{\mathbf{r}}_{f}^{(1)}}{m_{f}} \wedge \mathbf{B}_{\mathrm{mat}}, \\
& \ddot{\mathbf{r}}_{f}^{(3)}+\frac{1}{\tau_{f}} \dot{\mathbf{r}}_{f}^{(3)}=-\frac{e \mathbf{P}_{b}^{(3)}}{m_{f} \epsilon_{0}}-\frac{e \mathbf{P}_{f}^{(3)}}{m_{f} \epsilon_{0}}-\frac{e \dot{\mathbf{r}}_{f}^{(3)}}{m_{f}} \wedge \mathbf{B}_{\mathrm{mat}} .
\end{aligned}
$$

As usual, one sums over all the $N_{f}$ free electrons in the Fourier space, so the above equations become

$$
\begin{aligned}
& \mathbf{P}_{f}^{(1)}=\epsilon_{0} \chi_{f}^{(1)} \mathbf{E}-i \xi_{f} \mathbf{P}_{f}^{(1)} \wedge \mathbf{e}_{z}+\chi_{f}^{(1)} \mathbf{P}_{b}^{(1)}, \\
& \mathbf{P}_{f}^{(3)}=\chi_{f}^{(1)} \mathbf{P}_{b}^{(3)}-i \xi_{f} \mathbf{P}_{f}^{(3)} \wedge \mathbf{e}_{z},
\end{aligned}
$$

where $\quad \chi_{f}^{(1)}=\frac{\mathfrak{n}_{f} e^{2}}{m_{f} D_{f}(\omega) \epsilon_{0}}, \quad \xi_{f}=\frac{i e \omega B_{\text {mat }}}{m_{f} D_{f}(\omega)}, \quad \mathfrak{n}_{f} \equiv N_{f} / V$, and $D_{f}(\omega)=\left(-\omega^{2}-\frac{\mathfrak{n}_{f} e^{2}}{m_{f} \epsilon_{0}}+\frac{i \omega}{\tau_{f}}\right)$ is the response function for the free electrons.

The two coupled systems for the components $P_{f_{x}}^{(n)}$ and $P_{f_{y}}^{(n)}$ $(n=1,3)$ are solved using the expressions of $\mathbf{P}_{b}^{(1)}$ and $\mathbf{P}_{b}^{(3)}$ given in Eqs. (27) and (28). Finally, by writing $\alpha_{f}^{(1)}=\frac{\chi_{f}^{(1)}}{1-\xi_{f}^{2}}$, the first- and third-order macroscopic polarization density tensor for free charges may be written as

$$
\begin{gathered}
\mathbf{P}_{f}^{(1)}=\epsilon_{0} \alpha_{f}\left[\begin{array}{ccc}
1 & -i \xi_{f} & 0 \\
i \xi_{f} & 1 & 0 \\
0 & 0 & X
\end{array}\right] \mathbf{E} \\
+\epsilon_{0} \alpha_{b} \alpha_{f}\left[\begin{array}{ccc}
\left(1+\xi_{b} \xi_{f}\right) & -i\left(\xi_{b}+\xi_{f}\right) & 0 \\
i\left(\xi_{b}+\xi_{f}\right) & \left(1+\xi_{b} \xi_{f}\right) & 0 \\
0 & 0 & X
\end{array}\right] \mathbf{E} \\
\mathbf{P}_{f}^{(3)}=\epsilon_{0} \alpha_{f} \alpha_{b}^{\prime(3)}\left[\begin{array}{ccc}
\left(1+\xi_{f} \xi_{b}\right) & -i\left(\xi_{f}+\xi_{b}\right) & 0 \\
i\left(\xi_{f}+\xi_{b}\right) & \left(1+\xi_{f} \xi_{b}\right) & 0 \\
0 & 0 & X
\end{array}\right] \mathbf{E} .
\end{gathered}
$$

Let us stress that the first term in Eq. (29) is completely equivalent to the well-known conductivity tensor $[\sigma]$.

\section{Total response function}

We are now ready for providing analytical expressions for the diagonal and nondiagonal elements of the dielectric tensor defined by formula (26). By summing the contribution of Eqs. (27)-(30) and by defining $\alpha_{b}^{\prime(3)}=\alpha_{b}^{(3)}\left(E_{x}^{2}+E_{y}^{2}\right)$ one gets

$$
\begin{aligned}
\epsilon_{x x} & =1+\alpha_{b}+\alpha_{f}+\alpha_{f} \alpha_{b}\left(1+\xi_{b} \xi_{f}\right), \\
\epsilon_{x y} & =-i\left[\xi_{b} \alpha_{b}+\xi_{f} \alpha_{f}+\alpha_{b} \alpha_{f}\left(\xi_{b}+\xi_{f}\right)\right], \\
\epsilon_{x x x x} & =\alpha_{b}^{(3)}+\alpha_{b}^{(3)} \alpha_{f}\left(1+\xi_{b} \xi_{f}\right), \\
\epsilon_{x x x y} & =-i\left[\xi_{b} \alpha_{b}^{(3)}+\alpha_{b}^{(3)} \alpha_{f}\left(\xi_{b}+\xi_{b}\right)\right] .
\end{aligned}
$$

In order to obtain the numerical values of these functions one has to fix the values of the following parameters: $\omega_{0}, \omega_{p}$, $\tau_{b}, \tau_{f}, x_{b}, x_{f}, a$, and $B_{\text {mat }}$. The $\omega_{0}$ pulsation is associated to the energy band gap between the electronic bands $3 d$ and $4 s$ of Ni. Argyres in Ref. 26 gives the values $\hbar \omega_{0}=4 \mathrm{eV}$. The value of the plasma pulsation $\hbar \omega_{p}=4.87 \mathrm{eV}$ can be found in Ref. 38. The damping factors $\tau_{b}$ and $\tau_{f}$ for bound and free charge carriers may be fixed at $10^{-15}$ and $10^{-14}$ s. ${ }^{39,40}$ According to Refs. 36, 37, and 41 we have $x_{b}=0.4$ and $x_{f}=0.6$. The distance $a$ is calculated with the lattice parameter $a_{l}$ of $\mathrm{Ni}$ which crystallizes in a face-centered cubic (fcc) structure. By using the atomic mass $M_{\mathrm{Ni}}=58.69 \mathrm{~g} / \mathrm{mol}$, the mass density $\rho=8.902 \mathrm{~g} / \mathrm{cm}^{3}$, the Avogadro's number $\mathcal{N}_{A}$, and the relation $\rho=4 M_{\mathrm{Ni}} /\left(\mathcal{N}_{A} a_{l}^{3}\right)$ we determine $a_{l}=3.51 \times 10^{-10} \mathrm{~m}$. In a fcc structure the distance between two nearly neighbors is $a=\frac{\sqrt{2}}{2} a_{l}=2.48 \times 10^{-10} \mathrm{~m}$. The value of the effective magnetic field will be discussed in the next section. Finally, we have assumed that $m_{f}=m_{b}=m_{e}$. In this case, one can write $x_{f} \omega_{p}^{2}=\frac{\mathfrak{n}_{f} e^{2}}{m_{f} \epsilon_{0}}$ and $x_{b} \omega_{p}^{2}=\frac{\mathfrak{n}_{b} e^{2}}{m_{b} \epsilon_{0}}$.

\section{RESULTS}

\section{A. Study without spin-orbit interaction}

In this part we do not consider the SOI. By using Eq. (25) we first plot the curves $\theta^{\min }=\operatorname{Re}\left[\Phi\left(\mathcal{E}_{\mathrm{abs}}^{\min }\right)\right]$ and $\eta^{\mathrm{min}}=\operatorname{Im}\left[\Phi\left(\mathcal{E}_{\mathrm{abs}}^{\min }\right)\right]$ as functions of $B_{\text {mat }}$ obtained for the lowest value of the absorbed energy $\left(\mathcal{E}_{\mathrm{abs}}^{\min }=10^{-3} \mathrm{~mJ} / \mathrm{cm}^{2}\right)$. Then, the comparison is made with the experimental values given by the authors of Ref. 12: $\theta_{\exp }^{\min }=7 \mathrm{mrad}$ and $\eta_{\exp }^{\min }=$ $9.7 \mathrm{mrad}$. 


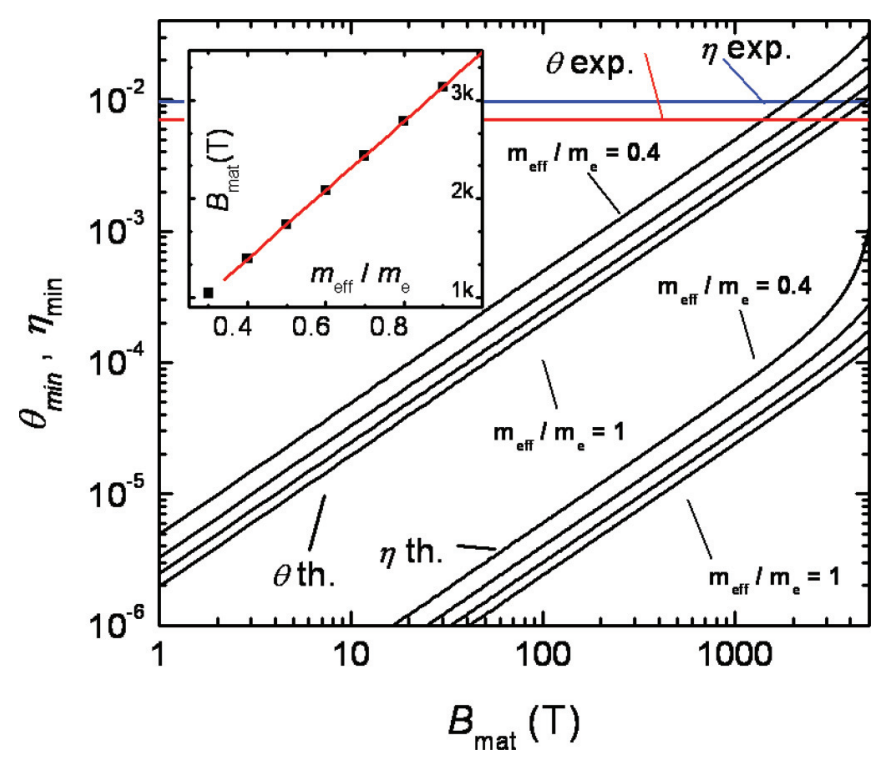

FIG. 3. (Color online) Faraday rotation and ellipticity at $\mathcal{E}_{\mathrm{abs}}^{\min }=$ $10^{-3} \mathrm{~mJ} / \mathrm{cm}^{2}$ (lowest value of the absorbed energy) as functions of the internal magnetic field $B_{\text {mat }}$ along with their experimental values taken from Ref. 12. Different values of the effective electron mass $m_{\text {eff }} / m_{e}$ are used: $0.4,0.6,0.8$, and 1 (by step of 0.2 ). Inset: value of $B_{\text {mat }}$ which matches the experimental value of $\theta^{\text {min }}$ as a function of $m_{\mathrm{eff}} / m_{e}$.

Due to the fact that nickel has a band structure, the calculation is also performed by substituting the electron mass $m_{e}$ by an effective mass $m_{\text {eff }}$. The results are presented in Fig. 3 for different values of $m_{\text {eff }} / m_{e}$ included in the interval $m_{\text {eff }} / m_{e} \in[0.3,1]$.

The first finding is that, with $B_{\text {mat }} \approx 10^{3} \mathrm{~T}$ (which is the order of magnitude of the Weiss molecular field in ferromagnetic materials), we recover the order of magnitude of the experimental value of the rotation parameter $\theta_{\exp }^{\min }$. Moreover, for decreasing values of $m_{\text {eff }}$, the intersection of the curves with $\theta_{\exp }^{\min }$ leads to smaller values of $B_{\text {mat }}$ (see the inset of Fig. 3). Let us mention that the tabulated value of $B_{\text {mat }}$ for nickel is $510 \mathrm{~T}^{26}$

Unfortunately, for the ellipticity parameter $\eta^{\text {min }}$, theoretical estimates are always much smaller in comparison to the experimental value. Indeed, the ellipticity parameter is related to the absorbtion in the material and cannot be perfectly accounted for within this classical model.

Normalized rotation $\theta / \theta^{\mathrm{min}}$ and ellipticity $\eta / \eta^{\mathrm{min}}$ as functions of the absorbed energy for different values of the effective electron mass are depicted in Figs. 4 and 5. For each value of $m_{\text {eff }}$, the associated internal magnetic field is determined using the results of the previous study performed at the lowest absorbed energy (we have chosen the results given by $\theta^{\mathrm{min}}$ which are more realistic than the ones given by $\eta^{\text {min }}$; see the inset of Fig. 3).

First of all, it can be clearly seen that the shape of the theoretical curves exhibits a nonlinear dependence very similar to the experimental one. Not only the shape but also the experimental values are reproduced for realistic parameters, namely, $m_{\mathrm{eff}} / m_{e}=0.3$. For both rotation and ellipticity, the relative change increases as the values of $m_{\mathrm{eff}} / m_{e}$ decrease,

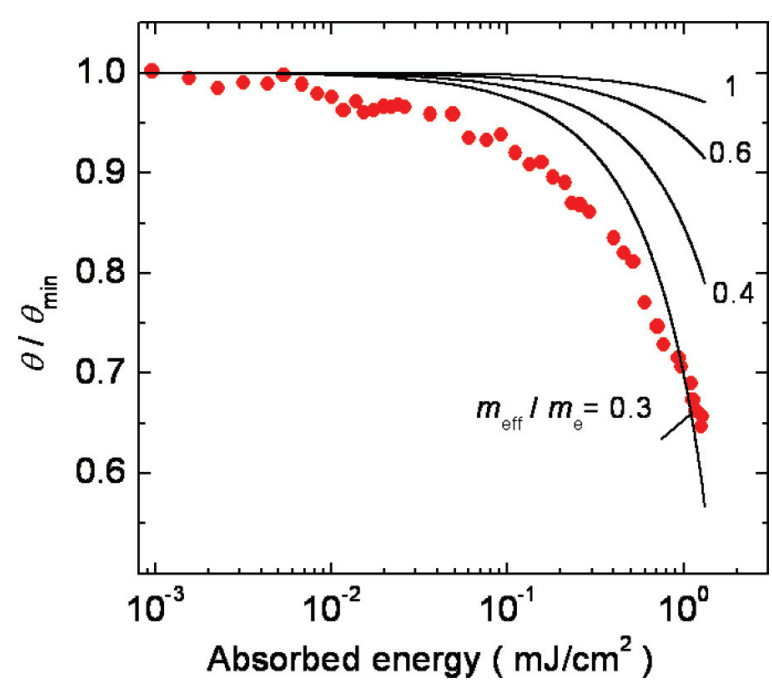

FIG. 4. (Color online) Normalized Faraday rotation for different values of the effective mass as a function of the absorbed energy. Full circles represent the experimental data taken from Ref. 12.

until going beyond the experimental result for $m_{\mathrm{eff}} / m_{e}=0.3$ (only valid for $\eta$ ).

In Fig. 6 we investigate the influence of the populations of bound and free electrons controlled by the values of $x_{b}$ and $x_{f}\left(x_{b}+x_{f}=1\right)$. Indeed, these quantities are only known approximatively. Three cases are considered $\left(x_{b}=0.38, x_{f}=\right.$ $0.62), \quad\left(x_{b}=0.40, x_{f}=0.6\right), \quad$ and $\left(x_{b}=0.42, x_{f}=0.58\right)$ with $m_{\mathrm{eff}} / m_{e}=1$.

\section{B. Study including the spin-orbit interaction}

Here we perform a similar study to that of the previous section but now incorporating the spin-orbit interaction by using a nonzero value of the magneto-optical parameter $\lambda=$ $\frac{i e M_{z}}{2 m c^{2} \omega \epsilon_{0}}$ in Eq. (24). In order to be compatible with our classical description, the parameter $\lambda$, which is a function of $M_{z}$, must be expressed as a function of the internal magnetic field $\mathbf{B}_{\text {mat }}$.

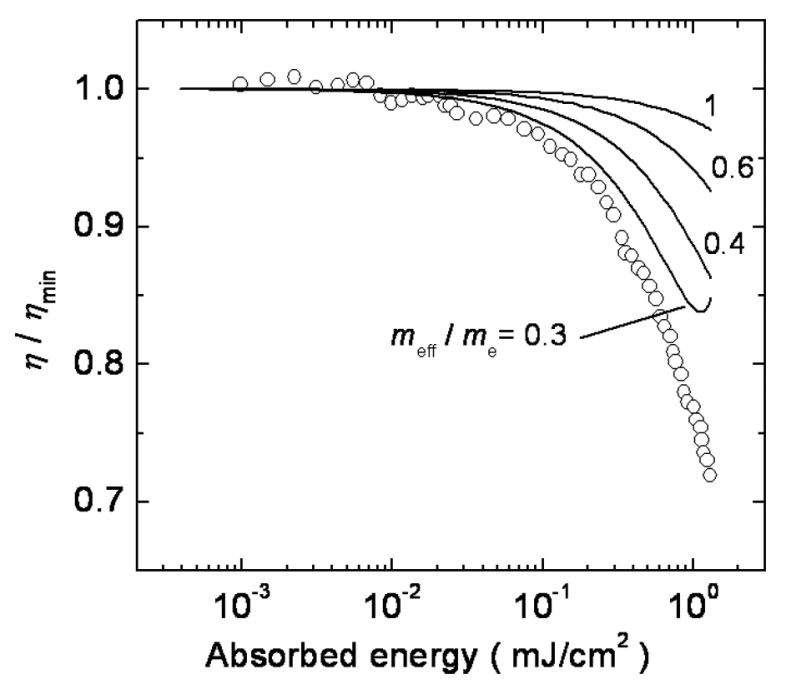

FIG. 5. Normalized Faraday ellipticity for different values of the effective mass as a function of the absorbed energy. Open circles represent the experimental data taken from Ref. 12. 


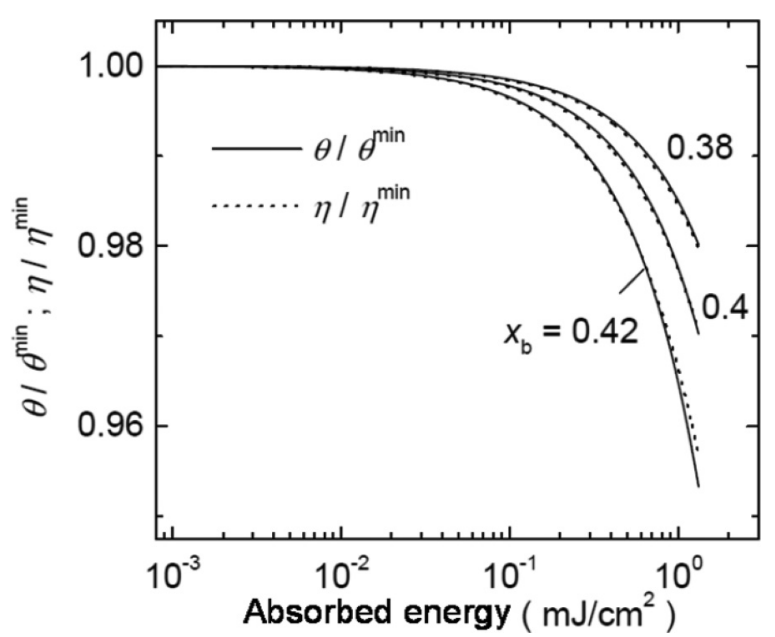

FIG. 6. Normalized Faraday rotation and ellipticity for different values of $x_{b}$ as functions of the absorbed energy. $m_{\mathrm{eff}} / m_{e}=1$.

By choosing the minimal relation $B_{\text {mat }}=\mu_{0} M_{z}, \lambda$ may be written as

$$
\lambda=\frac{i \omega_{B_{\mathrm{mat}}}}{2 \omega},
$$

where $\omega_{B_{\text {mat }}}=\frac{e B_{\text {mat }}}{m}$ is the cyclotron pulsation of the ferromagnetic material and $\omega$ is the pulsation of the external electric field. The magneto-optical parameter originating from the SOI thus quantizes the competition between these two pulsations. This modification incorporated into the optical indices given by Eq. (24) gives interesting results in comparison with the case where $\lambda=0$.

The result of the calculations of $\theta^{\min }=\operatorname{Re}\left[\Phi\left(\mathcal{E}_{\mathrm{abs}}^{\min }\right)\right]$ and $\eta^{\min }=\operatorname{Im}\left[\Phi\left(\mathcal{E}_{\text {abs }}^{\min }\right)\right]$ with $\lambda \neq 0$ as functions of $B_{\text {mat }}$ for the lowest energy $\left(\mathcal{E}_{\mathrm{abs}}^{\min }=10^{-3} \mathrm{~mJ} / \mathrm{cm}^{2}\right)$ are presented in Fig. 7 . Compared to Fig. 3, the intersection between the theoretical estimates of $\theta^{\min }$ and the experimental data $\theta_{\exp }^{\min }$ appears for

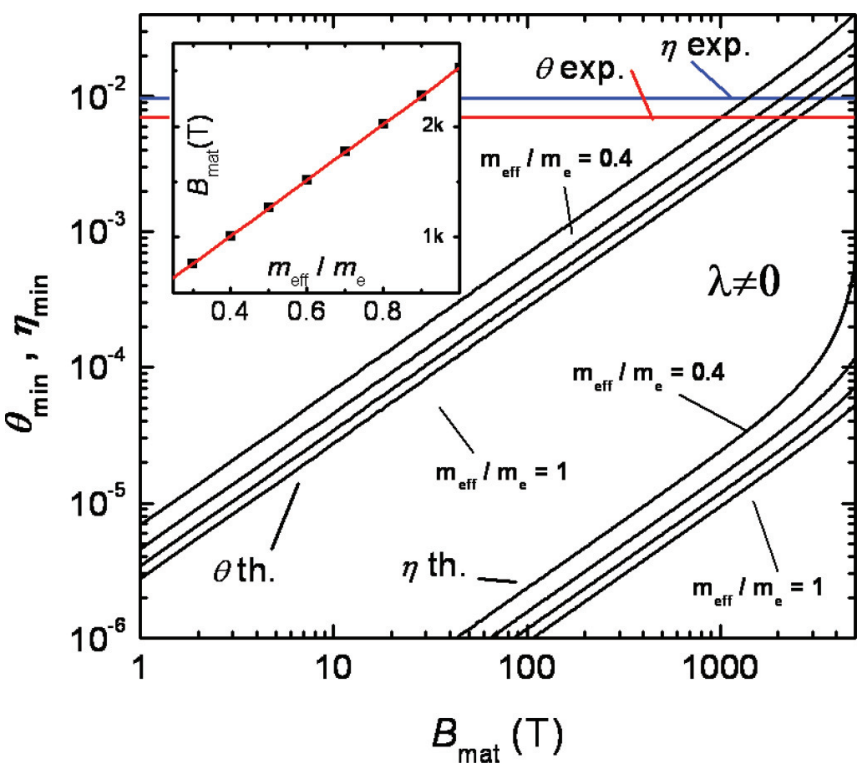

FIG. 7. (Color online) Faraday rotation and ellipticity at $\mathcal{E}_{\text {abs }}^{\min }=$ $10^{-3} \mathrm{~mJ} / \mathrm{cm}^{2}$ as a function of $B_{\text {mat }}$ with $\lambda \neq 0$ along with their experimental values taken from Ref. 12 .

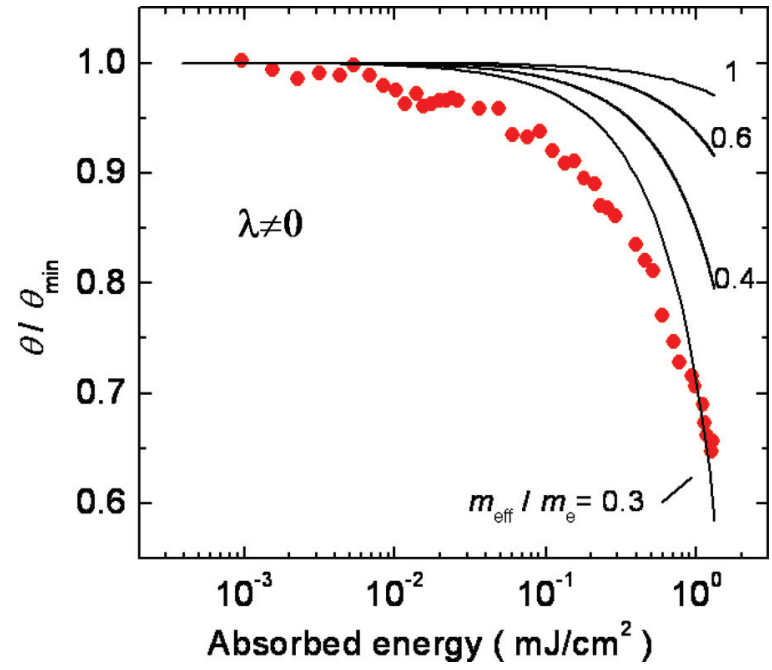

FIG. 8. (Color online) Normalized Faraday rotation for different values of the effective mass as a function of the absorbed energy with $\lambda \neq 0$. Open circles represent the experimental data taken from Ref. 12.

smaller values of $B_{\text {mat }}$. Therefore, one concludes that the incorporation of the SOI in the model leads to more realistic values for describing the effective magnetic field inside a nickel ferromagnetic sample. However, theoretical estimates of the ellipticity $\eta^{\min }$ are still really far from the measured value $\eta_{\exp }^{\min }$.

Using this new value of $B_{\text {mat }}$, the normalized rotation $\theta / \theta^{\mathrm{min}}$ and ellipticity $\eta / \eta^{\min }$ as functions of the absorbed energy and for different values of $m_{\mathrm{eff}} / m_{e}$ are depicted in Figs. 8 and 9. The evolution of $\theta / \theta^{\mathrm{min}}$ is shown in Fig. 8. It can be clearly seen that the results are very similar to those of Fig. 4 where $\lambda=0$. At first glance one is tempted to say that the SOI has no effect. This conclusion is of course wrong. Indeed two effects due to the SOI cancel each other. The first one is the decrease of the effective magnetic field imposed by the result at the lowest energy (see Fig. 7) and the second one is the modification due to $\lambda \neq 0$ of the optical indices given by Eq. (24).

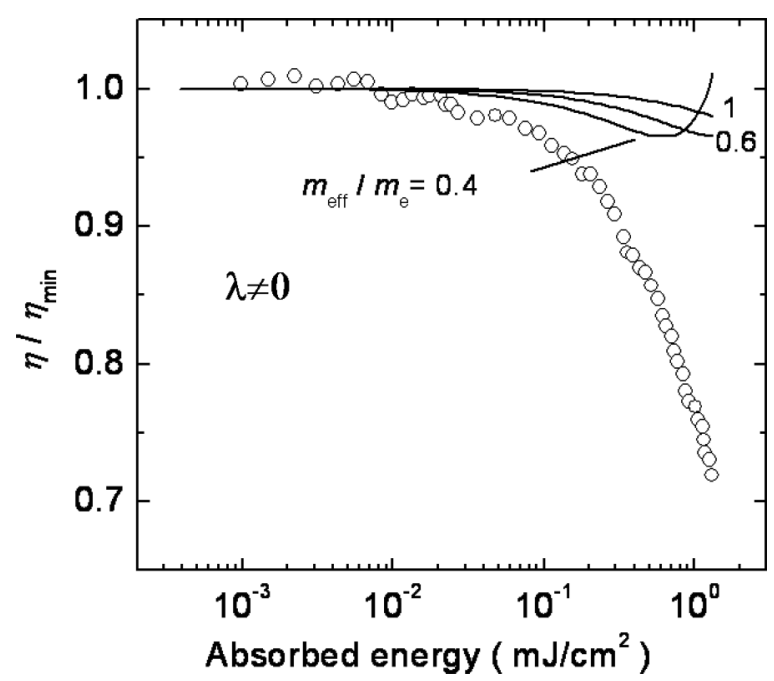

FIG. 9. Normalized Faraday ellipticity for different values of the effective mass as a function of the absorbed energy with $\lambda \neq 0$. Open circles represent the experimental data taken from Ref. 12. 
Finally, in Fig. (9) the relative change of the normalized ellipticity $\eta / \eta^{\mathrm{min}}$ as a function of the absorbed energy is depicted. One can clearly see that the theoretical estimations are in complete disagreement with the experimental measurements, being worse than the situation depicted in Fig. 5 where $\lambda=0$. Indeed, we have already emphasized that the ellipticity parameter which is related to the light absorption of the physical system seems to be difficult to model by using a classical framework.

\section{DISCUSSION AND CONCLUSIONS}

\section{A. Discussion}

Here, we discuss the advantages and the limits of this work and propose some ideas to improve the model.

The most important result is that the nonlinear shape of the theoretical estimates seems to follow the trend of the experimental results. Therefore this suggests that both Eqs. (18) and (24) are appropriate for describing a nonlinear anisotropic medium. The results obtained for $\theta^{\text {min }}$ and $\theta / \theta^{\text {min }}$ are in good agreement with the experimental values, especially when $\lambda \neq 0$ because, for this case, the needed value of $B_{\text {mat }}$ is more realistic. This confirms the crucial role played by the spin-orbit interaction in the Faraday effect, and Eq. (24) can also be seen as a new manner to incorporate SOI effects in a macroscopical description of magneto-optical phenomena.

Moreover, the modeling of $\eta^{\text {min }}$ and $\eta / \eta^{\text {min }}$ is unsatisfactory. The theoretical estimates are two orders of magnitude below the experimental value $\eta_{\exp }^{\min }$. We have described classically the absorption through the damping factors $\tau_{b}$ and $\tau_{f}$ and their values were fixed in the frame of linear optics. In such conditions involving nonlinear effects, a classical description seems to be clearly inadequate. The use of a classical framework was done on purpose for the sake of simplicity but one could improve the modeling by using quantum dielectric response functions. Let us also mention, that we have worked with only one mode $\omega$ of the electromagnetic field without considering the spectral distribution $\Delta \omega$ of the laser pulse. However, we strongly believe that the main conclusions will be not affected by this improvement of the model.

Another important comment concerns the magnetization dynamic which is missing in the present model. Indeed, the latter uses a "static" magnetization $B_{\text {mat }}=f(M)$ and the difference between the theoretical predictions and the experimental data could be associated to this missing ingredient. This interpretation leads to two interesting conclusions. First, the part of the magneto-optical signal calculated in Figs. 4 and 8 is thus mainly related to the nonlinear response of free and bound electrons, classically explained by a nonlinear distortion of their orbital momenta. Secondly, finding the appropriate value of $m_{\mathrm{eff}} / m_{e}$ could also help to fix the difference between the theoretical predictions and the experimental results.

Thus, we propose that the spin-orbit interaction and the charge motion influenced by the effective magnetic field have to be considered in the nonlinear regime. This idea must be put in contrast with the common belief that the effective magnetic field in a ferromagnetic material cannot affect the motion of charges. This model could be also improved by adding a magnetization dynamic (e.g., through the Bloch equations) and/or by calculating the dielectric response functions in a more rigorous way (e.g., using a quantum modeling).

\section{B. Conclusions}

Based on the classical anharmonic Drude-Voigt theory, a rather simple model is developed for modeling ultrafast nonlinear coherent magneto-optical experiments performed on ferromagnetic thin films. Theoretical estimations of the Faraday rotation angles are compared to available experimental values of nickel thin film and give meaningful insights about the physical mechanisms underlying the observed coherent magneto-optical phenomena. Under realistic conditions, the model successfully explains the observed trends. The crucial role played by the spin-orbit mechanism resulting from the direct interaction between the external electric field of the laser and the electron spins of the sample is underlined.

\section{ACKNOWLEDGMENTS}

Y.H. and P.-A.H. would like to thank J.-Y. Bigot and M. Vomir for providing us with their experimental data and for useful discussions. Y.H. also thanks H. Vonesch for her helpful remarks and advice. This work was supported in part by the project ATOMAG supported by the European Research Council Advanced Grant No. ERC-2009-AdG-20090325247452.
${ }^{1}$ E. Beaurepaire, J. C. Merle, A. Daunois, and J.-Y. Bigot, Phys. Rev. Lett. 76, 4250 (1996).

${ }^{2}$ J.-Y. Bigot, C. R. Acad. Sci., Ser. IV: Phys., Astrophys. 2, 1483 (2001).

${ }^{3}$ A. Kirilyuk, A. V. Kimel, and Th. Rasing, Rev. Mod. Phys. 82, 2731 (2010).

${ }^{4}$ J.-Y. Bigot and M. Vomir, Ann. Phys. (Berlin) 525, 2 (2013).

${ }^{5}$ B. Koopmans, G. Malinowski, F. Dalla Longa, D. Steiauf, M. Fähnle, T. Roth, M. Cinchetti, and M. Aeschlimann, Nat. Mater. 9, 259 (2010)

${ }^{6}$ M. Lisowski, P. A. Loukakos, A. Melnikov, I. Radu, L. Ungureanu, M. Wolf, and U. Bovensiepen, Phys. Rev. Lett. 95, 137402 (2005).
${ }^{7}$ G. Lefkidis, G. P. Zhang, and W. Hübner, Phys. Rev. Lett. 103, 217401 (2009).

${ }^{8}$ W. Hübner and G. P. Zhang, Phys. Rev. B 58, R5920 (1998).

${ }^{9}$ G. P. Zhang and W. Hübner, Phys. Rev. Lett. 85, 3025 (2000).

${ }^{10}$ C. Boeglin, E. Beaurepaire, V. Halté, V. Lopez-Flores, C. Stamm, N. Pontius, H. Dürr, and J.-Y. Bigot, Nature (London) 465, 458 (2010).

${ }^{11}$ M. Krauß, T. Roth, S. Alebrand, D. Steil, M. Cinchetti, M. Aeschlimann, and H. C. Schneider, Phys. Rev. B 80, 180407(R) (2009).

${ }^{12}$ J.-Y. Bigot, M. Vomir, and E. Beaurepaire, Nat. Phys. 5, 515 (2009).

${ }^{13}$ H. Vonesch and J.-Y. Bigot, Phys. Rev. B 85, 180407(R) (2012). 
${ }^{14}$ G. P. Zhang, W. Hübner, G. Lefkidis, Y. Bai, and T. F. George, Nat. Phys. 5, 499 (2009).

${ }^{15}$ W. Hübner and K. H. Bennemann, Phys. Rev. B 40, 5973 (1989).

${ }^{16} \mathrm{~K}$. H. Bennemann, Nonlinear Optics in Metals (Oxford Univerity Press, New York, 1995).

${ }^{17}$ P. M. Oppener and A. Liebsch, J. Phys. Condens. Matter 16, 5519 (2004).

${ }^{18}$ Yu. P. Svirko and N. I. Zheludev, Polarization of Light in Nonlinear Optics (Wiley, New York, 1998).

${ }^{19}$ G. Lefkidis and W. Hübner, Phys. Rev. B 87, 014432 (2013).

${ }^{20}$ J. Chovan and I. E. Perakis, Phys. Rev. B 77, 085321 (2008).

${ }^{21}$ M. D. Kapetanakis, I. E. Perakis, K. J. Wickey, C. Piermarocchi, and J. Wang, Phys. Rev. Lett. 103, 047404 (2009).

${ }^{22}$ M. D. Kapetanakis, P. C. Lingos, C. Piermarocchi, J. Wang, and, I. E. Perakis, Appl. Phys. Lett. 99, 091111 (2011).

${ }^{23}$ W. Voigt, Magneto- und Elektrooptik (B. G. Teubner, Leipzig, 1908).

${ }^{24}$ W. Voigt, Phys. Z. 16, 298 (1915)

${ }^{25}$ H. R. Hulme, Proc. R. Soc. London, Ser. A 135, 237 (1932).

${ }^{26}$ P. N. Argyres, Phys. Rev. 97, 334 (1955).

${ }^{27}$ H. S. Bennett and E. A. Stern, Phys. Rev. 137, A448 (1965).

${ }^{28}$ H. Le Gall, J. Phys., Colloq. 32, C1-590 (1971).
${ }^{29}$ G. Manfredi and P.-A. Hervieux, Opt. Lett. 30, 3090 (2005).

${ }^{30}$ For symmetry reasons (centrosymmetric systems) the third-order term is not considered.

${ }^{31}$ R. W. Boyd, Nonlinear Optics (Academic, New York, 2003).

${ }^{32}$ L. D. Landau and E. M. Lifshitz, Quantum Mechanics: NonRelativistic Theory (Butterworth-Heinemann, Oxford, 1977).

${ }^{33}$ P. Strange, Relativistic Quantum Mechanics (Cambridge University Press, Cambridge, 2005).

${ }^{34}$ Y. Hinschberger and P.-A. Hervieux, Phys. Lett. A 376, 813 (2012).

${ }^{35} \mathrm{~A}$ complete derivation of $\mathbf{j}^{(2)}$ can be found in: A. Dixit, Y. Hinschberger, J. Zamanian, G. Manfredi, and P.-A. Hervieux, Phys. Rev. A 88, 032117 (2013).

${ }^{36}$ C. Kittel, Introduction to Solid State Physics (Wiley, New York, 1996).

${ }^{37}$ C. Zener, Phys. Rev. 83, 299 (1951).

${ }^{38}$ Handbook of Optical Constants of Solids, edited by E. D. Palik (Academic, Orlando, 1985).

${ }^{39}$ S. N. Paul, S. K. Bhattacharya, S. Mondal, and B. Chakraborti, Fizika A 13, 161 (2004).

${ }^{40}$ L. B. Scaffardi and J. Tocho, Nanotechnology 17, 1309 (2006).

${ }^{41}$ M. A. Ordal, L. L. Long, R. J. Bell, S. E. Bell, R. R. Bell, R. W. Alexander, Jr., and C. A. Ward, Appl. Opt. 22, 1099 (1983). 